IZA DP No. 8860

Gender Effects, Culture and Social Influence in the Dictator Game: An Italian Study

Niall O'Higgins

Arturo Palomba

Patrizia Sbriglia

February 2015 


\title{
Gender Effects, Culture and Social Influence in the Dictator Game: An Italian Study
}

\author{
Niall O'Higgins \\ University of Salerno \\ and IZA \\ Arturo Palomba \\ University of Naples II \\ Patrizia Sbriglia \\ University of Naples II

\section{Discussion Paper No. 8860 \\ February 2015} \\ IZA \\ P.O. Box 7240 \\ 53072 Bonn \\ Germany \\ Phone: +49-228-3894-0 \\ Fax: +49-228-3894-180 \\ E-mail: iza@iza.org
}

\begin{abstract}
Any opinions expressed here are those of the author(s) and not those of IZA. Research published in this series may include views on policy, but the institute itself takes no institutional policy positions. The IZA research network is committed to the IZA Guiding Principles of Research Integrity.

The Institute for the Study of Labor (IZA) in Bonn is a local and virtual international research center and a place of communication between science, politics and business. IZA is an independent nonprofit organization supported by Deutsche Post Foundation. The center is associated with the University of Bonn and offers a stimulating research environment through its international network, workshops and conferences, data service, project support, research visits and doctoral program. IZA engages in (i) original and internationally competitive research in all fields of labor economics, (ii) development of policy concepts, and (iii) dissemination of research results and concepts to the interested public.
\end{abstract}

IZA Discussion Papers often represent preliminary work and are circulated to encourage discussion. Citation of such a paper should account for its provisional character. A revised version may be available directly from the author. 
IZA Discussion Paper No. 8860

February 2015

\section{ABSTRACT}

\section{Gender Effects, Culture and Social Influence in the Dictator Game: An Italian Study}

There is little consensus on whether women are more generous than men; some research results indicate a higher propensity towards giving of female dictators, whilst others suggest the opposite. Two explanations have been put forward. According to the first one, women are more generous than men and the conflicting results are due to the way preferences are elicited (Eckel and Grossman, 2002), since women are more sensitive to "social cues" and their preferences are more "malleable" (Croson and Gneezy, 2009). According to the second one, the institutional culture and the role women have in society are key elements in shaping gender differences in preferences. In fact, in matrilineal societies (Gong et al.; 2014; Gneezy et al.; 2009), women are self-oriented, more competitive and less generous than men, since they have an important role as economic decision makers in the family and the society. We test these alternative hypotheses running Dictators experiments in Italy, a western country with a matrilineal culture, introducing - at the same time - social influence in the design. We find more support to the hypothesis on the cultural role in shaping preferences, rather than the effects of social influence.

JEL Classification: $\quad$ C90, C91, D03, J16

Keywords: social influence, gender, social preferences, experiments, dictator game

Corresponding author:

Patrizia Sbriglia

Department of Economics

University of Naples II

Gran Priorato di Malta, Capua (CE)

Italy

E-mail: Patrizia.Sbriglia@unina2.it 


\section{Introduction}

The impact on behaviour of gender differences in preferences is an important topic of much relevance to economics and economic policy; evidence of such differences can be found in all forms of economic and social exchanges. Much research have been devoted to the evaluation and to the study of the causes of gender divergence in behaviour and choice. Experimental Economics has proved a useful instrument in this area, providing evidence on sex differences in both economic preferences and risk attitudes. Great attention has been paid to the evaluation of gender biases in social preferences, altruism and pro- social behaviour. The importance of the issue arises from the fact that many social environments which are economically relevant - such as the labour market and the provision of public goods - are affected by individuals' preferences including their attitudes towards giving, trusting and cooperating.

One influential result in this specific field is that women are more pro-socially oriented than men, less selfish and more cooperative. Quoting C. Darwin ("women are tender and men are ambitious..."), Eckel and Grossman, 1998, find that women donate twice as much as male dictators. Similar results have been found in several replications of Dictator Game experiments (see Handbook of Experimental Economics results, 2008a, Croson and Gneezy, 2009), however, a consensus is lacking on the general applicability of the result. Indeed, the finding has so often been contradicted (see Croson and Gneezy, 2009, for an extended review) that a number of possible explanations for the opposite view have also been put forward.

One important possible explanation for the contradictory findings is that sex differences in social preferences are particularly sensitive to the way such preferences are elicited (Croson and Gneezy, 2009; Eckel and Grossman, 1998). For example, as far as altruism and generosity (in the dictator game) are concerned, sex differences are found in experiments where choices are elicited in a totally anonymous context, but the evidence is much less clear cut if the condition of anonymity is relaxed.

In choices where generosity and inequity aversion play a role (the ultimatum game), there are similar results but with a further complication. In any strategic context, in fact, choices are affected by strategic uncertainty; therefore, observed behavioural differences due to differences in risk attitudes may erroneously be attributed to differences in individuals' social preferences, 
(Eckel and Grossman, 2008a); the extent to which men and women's choices differ may not be separately identifiable.

The fact that sex differences are identified in some specific contexts but disappear (or become negligible) in others suggests that women and men perceive and respond to sociality - or social cues, social network composition, social pressure, etc. - in a different way, and that the measurement of the difference in their social behaviour is affected by the context in which they are operating. Thus, there are difference in social preferences, however, the different response of men and women to social relationships may confuse the results of the investigations.

Both experimental and psychological research on gender differences have provided strong support to the hypothesis that women are much more sensitive than men to "social cues". Furthermore, measuring the impact that the observation of peers' actions may produce on behaviour is in itself a key element in understanding how women and men respond to social relationships and adapt to the observed rules of conduct in social groups and networks, therefore the topic has attracted much attention in both fields.

To take some examples, Kettner and Ceccato (2014) interpret as socially relevant the sex pairing in the dictator game, arguing that women respond differently to women co-players than to men co-players. ${ }^{1}$ Similarly, Balliet et al. (2011) conduct a meta-analytic review of sex differences in cooperative behaviour, concluding that women are more cooperative in some contexts, less in others, compared to men. This depends on the different response to social relationships and groups. Women tend to be kinder and cooperative to friends and family, men are more cooperative to strangers and in larger groups. Also, the gender composition of the social group may play a role in shaping the difference. Balliet et al. (2011) report that women are more cooperative in all-female groups; however, the same evidence is not found in allocation models (Dwefenberg and Muren; 2006).

A further element of difference in the way men and women react to social relationships and contexts, is provided by the experimental evidence on the effects' of peers influence. Charness and Rustichini (2010) examine women and men's behaviour in social dilemma games where choices are made publicly under the scrutiny of an external party of peers. Women tend to be more sensitive to peer pressure and to be more cooperative when their choice is being observed.

\footnotetext{
${ }^{1}$ Specifically, they argue that women tend to be more competitive and less altruistic when facing women. They quote a number of papers corroborating this point of view.
} 
Women and men also differ in the perception of what can be regarded as "socially fair behaviour" and on their willingness to conform to it. In Della Vigna et al. (2013), the authors find that that women tend to be as generous as men in a door-to-door solicitation, but controlling for the solicitors avoidance, then the female's level of altruism is significantly reduced.

The hypothesis that women tend to conform to socially fair behaviour is supported by the results of a modified Dictator Game (Heinz et al., 2012) where respondents move first and gain the endowment on which the Dictator have then to decide the final allocation. The experiments consist of three different designs: "the real effort design", where respondents gain the endowment, "the windfall design" where the endowments are randomly allocated to respondents; "the lottery design" where endowments are allocated to respondents according to a lottery. The authors find that female dictators allocate higher shares of the endowment in the real effort design, particularly to respondents who worked hard to gain the final pot. On the contrary, they do not find significant differences in male dictators' donations across treatments. Overall, they find that women dictators tend to be more generous and men tend to be more selfish. ${ }^{2}$

Recently, field experiments have provided an important contribution to the debate on gender differences in pro-social behaviour, by taking a completely different perspective on the issue and achieving very innovative results.

Specifically, Gong et al.; 2014, and Gneezy et al.; 2009, have conducted field experiments in different societies in China, Tanzania and India. The distinguishing trait of these ethnical groups lies in the position women have in the community and the relevance of their roles as economic decision makers in family life.

Specifically, Khasi in India and Masuo in China are matrilineal societies, women manage the family income and take economically relevant decisions. Yo in China and Masaai in Tanzania are patriarchal societies and the roles of men and women are reversed. The authors study gender differences in altruism and propensity to compete. Their results are striking: in societies where women have an important role in the economic life, they are less altruistic and more competitive than men.

\footnotetext{
${ }^{2}$ Also, men and women differ on the expectations they have on the outcome of social exchanges. Focusing on the dictator game, Rigdon and Levine (2011), find that when the expectations on the other's giving are homegrown, then there is a substantial difference in men and women altruistic behavior, however, when expectations of receiving are uniform, differences disappear. Aguilar et al.; (2009) find that women expect women to be more generous while men believe there are no differences. Both papers open an interesting field of investigation, since they claim gender differences in behavior may arise because men and women have different expectations on how men and women will act socially.
} 
To be precise, Mauso women are more selfish than Masuo men. Differences in altruism are negligible in the Yo society, a patriarchal society, where men and women have however similar roles in the family. Studying competitiveness, the results are clear cut. In the Khasi group, women are more competitive than men. Findings totally reversed in the Masaai ethnical group, where Masaai men showed a propensity to compete double that of Masaai women. The interesting point raised by these results is that there might be a missing factor in the analysis of gender differences related to the importance of the institutional culture of the society under investigation. Perhaps these findings be drawn upon to explain and compare the existing contributions on gender differences?

As stated in Gong et al.; 2014, most analyses on altruism which claim a higher female propensity to donate and to be selfless have been conducted in Western countries (US, Europe), which have a patriarchal culture and where the role of the women is much less connected to the economic life.

Thus, more than innate differences that may be confounded by social contexts or the way preferences are elicited, it may be reasonable to suppose that the patriarchal culture is playing a determining role, and that this makes women appear more tender and men more ambitious.

The experiment presented in this paper aims at providing a test to these alternative (but not mutually exclusive) points of view on gender differences in altruism.

We conducted a Dictator experiment in Southern Italy - a Western society with a profound matrilineal culture - and we introduce social influence in some sessions, in order to study whether women are more sensitive to social stimuli. In two out of the three sessions, in fact, we gave the dictator an additional piece of information, communicating how much was donated by a dictator in a different session and, in one of these sessions, also the dictator's gender.

Our final goal is to test whether culture is more important in this context than social influence, and whether female preferences are more malleable than male preferences. If culture is more important than social influence, we would expect that the reaction to the signal will be indistinguishable between men and women, and we also expect women to be significantly less generous than men.

Our results are rather striking. Analyzing the donations of 90 dictators distributed over the three sessions which constituted our experiment, we find that our dictators are - overall - rather stingy, but women are significantly less generous than men. 
We also find that both male and female dictators' donations are affected by social influence but there is no significant difference in the relative importance men and women give to the signal. However, results are more nuanced, since both genders give more importance to signals coming from dictators of the same sex, and whilst men are relatively more sensitive to social influence, the impact of the signal is greater for women.

Thus, we find that it is important to take into account the fact that the experiments were run in Southern Italy with its specific cultural context.

In fact, Italy, and especially Southern Italy, is a context in which the matrilineal culture still persists and where the role of the woman in the household is centered on managing the family finances and the role of the man is more socially oriented. Thus - as stereotypes on Italian men often suggest - women are more responsible decision makers when it comes to financial affairs, and, as a rule, young men leave the family home and take financial independent decisions relatively late in life compared to young men born into other European societies. As is the case also in Chinese culture, even when kids leave the family and live independently, the relationships with parents is so close that mothers intervene in the financial decisions also of the children' families.

In order to sketch an explanation of the origin of the matrilineal culture, it is important to recall that the specific territory where the University of Naples II is located - Caserta - is an area in which the Roman cult of the Matres Matutae goddess flourished and where the world's most important Museum on the Matres Matutaes stands. The origin of this specific cult is to be found in pre-Catholic and pagan Italian culture. The cult of the Matres Matutae (Aurora) was centered on the leading role of the woman in society and in the family, since women had the primary gift of fertility. Specifically, women were in charge of children's education and child bearing; and the villages survival chances were strictly connected to the strength and to the number of soldiers and fighters. All these activities gave women a prominent role in their social groups. The myth was annulled with the advent of Catholicism but many features of that culture still dominate Southern Italian societies. ${ }^{3}$

\footnotetext{
${ }^{3}$ Before the Catholic advent, also a different myth - of Greek origin - had a strong impact on Southern Italian culture, which gave importance to masculinity rather than femininity and it was represented by Priapus, whose representation can be found in the Pompei remains. However, though, the Greek Priapus was a fertility deity, as Mates Matutae, in the Roman culture, Priapus received attention only in paintings and sculptures of erotic arts. One might observe in passing that, in modern times, the importance of female fertility as a basis for their primacy has found support in Ashley Montagu's eloquent defence of the 'Natural Superiority of Women' in his homonymous book (Montagu, 1953); Montagu's thesis is centred precisely around the ability of women to bear children.
} 
The paper is organized as follows. In section 2 we describe the experimental design and state the research hypotheses; we also locate our experiment and predictions in the vast literature on gender and altruism. In section 3 we report the results of the data analysis, which helps us to provide answers to the behavioural questions. Section 4 concludes.

\section{Experimental design and procedures}

We implemented a modified version of the Dictator Game experiment presented in Eckel and Grossman, 1998 and Hoffman et al.; 1994. The most important aspect of these designs is that they preserve the total anonymity of individuals' choices (double blind). Dictators decide on the allocation in a condition of total isolation from other dictators and respondents, as well as experimenters. As stated in the introduction, if women and men respond differently to social cues and peers' pressure then anonymity is an important factor to measure distances between female and male donations, when social influence is introduced.

We conducted three sessions of the experiment, involving 180 subjects. All sessions were run on a single day, participants were recruited in all departments of the University and great care was devoted to check that dictators and respondents were separated and unable to communicate. ${ }^{4}$ Dictators could see that respondents were taken to a different room, and they were seated separately from each other. They received an envelope containing two sheets of papers, one for the instructions and one to indicate the player's sex.

In all sessions, only the dictator's number was reported on the envelopes (A11, $A 12$, etc. for session 1; $A 21, A 22$, etc.; for session 2; $A 31, A 32$, etc.; for session 3 ) and subjects learnt their role only when reading the Instructions sheet. In sessions 2, however, in addition to the instructions and the indication of the sex, dictators could view the choice a randomly chosen dictator of session 1 , and in session 3 they received also the information on the dictator's sex. Both pieces of information were contained in a small envelope enclosed in the large one.

\footnotetext{
${ }^{4}$ We also tried to enroll dictators and respondents in different colleges, to make sure that they had not met before. The experimental sessions took place in May 2014.
} 
At the beginning of each session, one student was randomly selected in both rooms to act as "monitor". The monitor earned a fixed amount of money, corresponding to the maximum earning of dictators. Her role consisted in forming random couples of participants and calculating the final payoffs. Upon arrival, all subjects received a participation fee of two euro, whilst the dictator's endowment was constituted of twenty experimental tokens (with an exchange rate of $€ 0.40$ per token). The endowment was reported in the Instructions sheet (see Appendix 1) and the dictator had to indicate on the same sheet how many tokens he or she wished to donate to the unknown respondent.

Once couples were formed, both monitors communicated that the experiment was over and that both dictators and respondents would collect their earnings the day after at the central secretarial student office of the University. The monitors were there checking that the individuals' payments were conducted efficiently and without mistakes.

Our design differs from the original double blind procedure in as much as subjects did not receive actual money in the envelope, but just the indication of the total number of tokens. Whilst the presence of banknotes and coins in the envelope increases realism, in our setting, it would have decreased the realism of the message delivered to dictators of sessions 2 and 3, since such information would have to be delivered to respondents either by monitors or by experimenters. The purpose of our analysis is to evaluate different responses to social influence of men and women, therefore we preferred to enhance the effect of the information. ${ }^{5}$ Table 1 sums up the main experimental aspects and the composition of the sample.

Table 1a: Dictators' distribution by gender

\begin{tabular}{|l|l|l|l|}
\hline & Session 1 & Session 2 & Session 3 \\
\hline & & & \\
\hline Male & 20 & 17 & 21 \\
\hline Female & 10 & 13 & 9 \\
\hline
\end{tabular}

\footnotetext{
${ }^{5}$ Our experimental design is similar in its procedural aspects to Servatka (2009), in as much our design adopts a between subjects' protocol; however, we replicate Eckel and Grossman (1998)' experiment without role reversal as in Servatka, where each subject plays in both roles (dictator and respondent).
} 
Table 1b: Dictators' distribution by Department ${ }^{6}$

\begin{tabular}{|l|l|l|l|l|l|}
\hline & Economics & Psychology & Biology & Maths & Politic \\
Science
\end{tabular}

\subsection{Research Questions}

The analysis of the experimental evidence of Session 1 will help us to understand whether cultural and historical elements may produce a different result on women generosity, or, as in previous replications of the Dictator Game in Western countries, Italian women are more generous than Italian men. Hence,

Research Question 1 (RQ1): Which is the fairer sex? Are Italian women more generous than Italian men?

We are also interested in identifying the effects of social influence on men and women's choices, therefore we are interested in providing answers to the following research questions,

Research Question 2 (RQ2): Do men and women respond to social cues in the Dictator Game?

But perhaps more importantly,

Research Question 3 (RQ3): Are women more sensitive to social influence than men?

\footnotetext{
${ }^{6}$ We conducted a Kolmogorov-Smirnov and an Epps-Singleton test to verify whether the high proportion of students reading Economics might have affected the results, determining an overall selfish behavior from Dictators in all sessions. Both tests rejected the null hypothesis of significant differences between Economic students and students coming from other departments.
} 
If so, are women conformist, do they adapt their behaviour to what the signal is indicating (i.e.; higher generosity if peers' actions signal high levels of altruism)? Or do women react to the information by moving in the opposite direction?

Finally, many studies have found that an important aspect of the gender differences in behaviour is related to the sex composition of the social group under investigation, and to the sameldifferent sex type of social exchanges. Here, the evidence collected in session 3 allows us to explore a novel aspect of this problem, e.g.; whether women (men) are more sensitive to peers' influence when they are observing the actions of an individual of the same or the opposite sex. Indirectly - if there exists a different response - we are claiming that women (men) trust the information they gain more (or less) when it comes from a woman (man). We therefore state the final issue to be resolved,

Research Question 4 (RQ4): Do women (men) trust information more if it comes from a female (male) dictator?

\section{Data analysis}

Table 2 allows a first examination of the issue of the research Questions. In the first session with no information, women are clearly less generous than their male counterparts and this difference - and its statistical significance - falls as information is introduced in sessions 2 and, above-all session 3 . Indeed the gender difference is only statistically significant (at $10 \%$ ) in the first session. Gender differences are also reflected in the distribution of tokens sent; only 5 (i.e. $6 \%$ ) of the 90 dictators sent over one half of their allocation and these were all men. Moreover, a further 13 dictators sent exactly half their allocation, of these 11 were men. Thus the prevalence of 'fair' or 'generous' dictators was $28 \%$ - over one in four - amongst men, but only $6 \%$ - less than one in ten - amongst women. 
Table 2: Mean no. of tokens sent by gender and session

\begin{tabular}{|l|c|c|}
\hline & Males & Females \\
\hline Session 1 (no information) & 7.65 & 5.40 \\
\hline $\begin{array}{l}\text { Session 2 (information on } \\
\text { amount sent) }\end{array}$ & 6.88 & 5.23 \\
\hline $\begin{array}{l}\text { Session 3 (information on } \\
\text { amount sent and gender of } \\
\text { sender) }\end{array}$ & 6.29 & 6.33 \\
\hline
\end{tabular}

The table provides a clear answer to Research question 1. The difference between male and female donations in session 1 is clearly statistically significant; in the context of no additional information, women are clearly stingier than men. It also is suggestive on the other questions in that the change in behaviour observed for both women (upwards) and men (downwards) provides some initial support for the idea that information is indeed playing a role and that this role differs for men and women.

Looking at this a little more formally, one finds that in sessions 2 and 3, the number of tokens sent is moderately (and similarly) correlated to the information provided to both men ( $r=$ 0.23 ) and women $(r=0.25)$. If we consider only session 3 , the correlation between female behaviour and the observed donations of other female dictators jumps to 0.5 , whilst the correlation between female behaviour and information falls practically to zero $(r=0.03)$ when this information concerns male donations. That is, women appear to respond much more readily to information on the tokens sent when this information concerns persons of their own gender.

Another way of looking at this is through the distance between information and donations. Table 3 reports the mean absolute and squared distance between male and female donations and the information on donations received by participants in the different situations. It will be observed that the distance between information and donations is always less for females than males. A second observation is that, looking at session 3 information according to the gender of the dictator and the gender of the person on whom information is provided - that is comparing the last two rows of table three - the gender 'bias' in the relevance of information begins to emerge. That is, the divergence between male information and male behaviour, and between female information and female behaviour is always less than the corresponding distance across 
genders. The substantial divergence in the mean squared difference for females according to whether the information provided regards the donations of men or women indicates also that women are particularly unified in their adherence to signals coming from female behaviour, whilst much less so when male behaviour is observed - indeed this is why the correlation coefficient is relatively high in the former case. Thus, with regard to research questions 3 and 4 above, the table suggests that indeed women are more sensitive to social information than men and that for both sexes, but particularly for women, individuals are more responsive to signals coming from persons of the same gender as opposed to from those of the opposite sex.

Table 3: Mean absolute and squared distances between information on donations and dictator donations, by gender

\begin{tabular}{|c|c|c|c|c|}
\hline \multirow{2}{*}{ Information } & \multicolumn{2}{|c|}{ Absolute difference } & \multicolumn{2}{c|}{ Squared difference } \\
\hline & Males & Females & Males & Females \\
\hline Session 2: tokens sent & 2.53 & 2.08 & 13.12 & 12.23 \\
\hline Session 3: tokens sent & 3.52 & 2.89 & 19.81 & 16.89 \\
\hline Session 3: tokens sent by males & 3.22 & 3.17 & 15.89 & 20.50 \\
\hline Session 3: tokens sent by females & 3.75 & 2.33 & 22.75 & 9.67 \\
\hline
\end{tabular}

Finally, the results reported in Table 4 allow us to take the analysis one stage further. The table reports the results of running simple Poisson regressions of the number of tokens sent in each of the three sessions as a function of the gender of the dictator and the information provided. The use of the Poisson model is preferred to OLS or tobit regressions since the dependent variable, the number of tokens sent, is a positive (or zero) integer and thus a count data model is a more appropriate approach, although the estimation of either of these models produces results which are qualitatively very similar to those reported here ${ }^{7}$.

\footnotetext{
${ }^{7}$ See, for example, Greene (2011) for a discussion and/or Cameron \& Trivedi (1998) for more details on count data models. As regards the Tobit model, note that there is only one lower limit observation in the sample and dictators never sent their entire allocation. Given this, the parameter estimates are almost identical for Tobit and OLS models.
} 
The first column of the table reports the results of a model including only dummy variables for gender which was run for dictators participating in the first, no information, treatment. The second column reports results for the second session where participants were given information on the tokens sent by another player. Here the reaction of male and female dictators is separately identified. In the final column the results the third session are reported. Here players were informed about the choice of another player and whether that person was male or female. This allows us to separately identify the reactions of males and females to the information also distinguishing the reactions to persons of the same or different genders.

Table 4: Poisson model of tokens sent

\begin{tabular}{|c|c|c|c|}
\hline & Session 1 & Session 2 & Session 3 \\
\hline & (No info) & $\begin{array}{c}\text { (Information on } \\
\text { tokens sent) }\end{array}$ & $\begin{array}{l}\text { (Information on } \\
\text { tokens sent and } \\
\text { gender of sender) }\end{array}$ \\
\hline Male & $1.93 * * * \quad(.092)$ & & \\
\hline - $\quad$ Tokens sent & & $0.19 * * *(.010)$ & \\
\hline By Males & & & $0.25^{* * *} \quad(.018)$ \\
\hline By Females & & & $0.18 * * * \quad(.013)$ \\
\hline Female & $1.65^{* * *}(.121)$ & & \\
\hline - Tokens sent & & $0.18 * * *(.015)$ & \\
\hline By Males & & & $0.16 * * * \quad(.017)$ \\
\hline By Females & & & $0.21 * * * \quad(.025)$ \\
\hline$n$ & 30 & 30 & 30 \\
\hline Log-Likelihood & -69.68 & -105.32 & -117.95 \\
\hline Wald test ( $p$-value) & .000 & .000 & .000 \\
\hline
\end{tabular}

The results first of all confirm that women are less generous than men in the no information treatment. The difference between men and women is substantial and is statistically significant (at $p<.05$ ). In the second treatment (column 2 ), both males and females react strongly 
to the information on another player; there is a weak suggestion that males react more strongly than females although the gender difference is clearly not statistically significant.

In the third treatment, once again it would appear that men react more strongly to information on other players than do women, although again the difference is not statistically significant. More importantly, however, the gender of the player whose information is being shared is important as is that of the recipient of the information. Specifically, males react more strongly to information on male players than they do for information on female players. For females, the reaction is similar but reversed. Women react more strongly to information when it comes concerns a female (as opposed to male) player. For men the difference in the reaction to information according to the gender of the source player is statistically significant $(p<.01)$ whilst for women this difference is not statistically significant at conventional levels $(p=.15)$. However if we jointly estimate the difference between the reaction of men and women to the same gender as compared to a different gender, this difference in reaction is clearly statistically significant $(p<$ $.01)$.

Taken together the results provide to basis for responding clearly to the various research questions, although there is a slight divergence in the results between the analysis of correlation and descriptive statistics on the one hand and the econometric analysis reported in table 4 on the other. The former suggest a closer relation between behaviour and information for females, whilst the latter suggests a (slightly) larger response to information for males than females. Literally interpreted, this suggests that women are more likely than men to react to social information, the male reaction to such information, where it occurs, is more substantial. We believe however, that it is fairer to conclude that the evidence presented here does not allow us to determine whether men or women are more reactive to social signals.

Thus, returning explicitly to the research questions, the evidence on RQ1 is unequivocal - in Italy, men are the 'fairer sex'; in the absence of additional information, men give more than women and they are more likely to behave fairly (dividing the allocated sum equally) or even altruistically (giving more to the recipient than they keep for themselves) than women are.

Similarly, regarding RQ2, the evidence presented here clearly supports the notion that both men and women respond to social signals; moreover, both sexes attach more weight to signals coming from members of the same sex (RQ4). On research question 3 , however, the results are more mixed and nuanced; on the one hand, however, the correlation between women' plays and information on other women is relatively high $(r=0.5)$ and the distance between the two is 
relatively low - that is, women's react more closely than men do to information on other women, or either do on other men - on the other hand, the size of the impact is (slightly) smaller for women than for men. Thus, it is best to conclude that the evidence does not allow a clear answer to this question.

\section{Concluding remarks}

This paper has tried to provide answers to some important question related to gender differences in altruistic behaviour.

Firstly, we try to provide a preliminary response to the question as to whether Italian women are more generous than Italian men. Secondly, we address the issue of the relative importance of social cues compared to cultural factors in shaping gender differences in preferences. Finally, on the specific point of social influence, we address the question of whether the sex of the observed dictator plays a role in defining the importance that male and female dictators give to social influence.

Our results are clear cut. Women are evidently not the fairer sex in the Italian context, and both men and women alter their behaviour in response to social cues. Moreover, both men and women react more to social signals coming from others of the same sex. Whether the impact of social influence is greater for women is less clear. Women's behaviour more closely mirrors social signals particularly when it comes from other women, however the measured size of such effects where they occur is slightly larger for men than for women.

Our results thus support the notion that culture is more important than social influence in determining differences across the findings of previous work on gender differences in social preferences. Certainly, cultural factors appear to moderate the specific effects of social influence. However, much more evidence in this area is needed to confirm or modify this result.

Finally, we want to underline the usefulness of our investigation for the connected area of research centered on the study of the effect of social influence on behaviour. In fact, our experiment is suggestive of important aspects of gender differences in behaviour in economically significant social situations in the real world. Women and men appear to attribute similar weight to social information, however, a special role is played by the gender of the observed peer: in fact, both men and women seem to value signals more if they come from subjects of their same 
gender. Therefore, due to the greater importance of same sex social signals, peer comparisons will tend to produce faster convergence of behaviour in groups and networks comprising a substantial majority of one sex, as opposed to mixed groups. 


\section{References}

Aguiar, F., P. Brañas-Garza, R. Cobo-Reyes, N. Jimenez and L. M. Miller, "Are women expected to be more generous?" Experimental Economics, 12: 93-98

Andreoni, J., and L. Vesterlund, 2001. "Which Is the Fair Sex? Gender Differences in Altruism." Quarterly Journal of Economics, 116(1): 293-312.

Balliet, D., S. J. Macfarlan, P. N. Li, M. Van Vugt, 2011, "Sex Differences in Cooperation: A Meta-analytic Review of Social Dilemma" Psychological Bulletin, vol. 137, n. 6, pp. 881-909.

Bolton, G. E. and E. Katok, 1995. "An Experimental Test for Gender Differences in Beneficent Behaviour." Economics Letters, 48(3-4): 287-92.

Cameron, A. C., and P. K. Trivedi, 1998. Regression analysis of count data. Cambridge University Press, Cambridge.

Cason T. and V. L. Mui, 1998. "Social influence in the sequential dictator game". Journal of Economic Psychology, 42, 246-265

Charness, G. and A. Rustichini, 2011. "Gender differences in cooperation with group membership", Games and Economic Behaviour, v. 72, Issue 1, 77-85.

Croson, R. and U. Gneezy, 2009. Gender Differences in Preferences, Journal of Economic Literature, 47:2,

Della Vigna, s., J. A. List, U. Malmendier and G. Rao, 2013. "The Importance of Being Marginal: Gender Differences in Generosity" NBER working papers, Febraury 2013.

Dufwenberg, M. and A. Muren. 2006a. "Gender Composition in Teams." Journal of Economic Behaviour and Organization, 61(1): 50-54.

Eckel, C. C. and P. J. Grossman, 1998. "Are Women Less Selfish than Men? Evidence from Dictator Experiments." Economic Journal, 108(448): 726-35.

Eckel, C. C. and P. J. Grossman. 2008a. "Differences in the Economic Decisions of Men and Women: Experimental Evidence." In Handbook of Experimental Economics Results, Volume 1, ed. Charles Plott and Vernon Smith, 509-19. New York: Elsevier.

Gneezy, U., K. L. Leonard and J. A. List, 2009. "Gender Differences in Competition: Evidence from a Matrilineal and a Patriarchal Society" Econometrica, Vol. 77, No. 5, 1637-1664

Gong, B., H. Yan and C.-L. Yang 2014, "Gender differences in the dictator experiment: evidence from the matrilineal Mosuo and the patriarchal Yi" forthcoming Experimental Economics

Greene, W. H. 2011, Econometric Analysis, 7th Edn, Prentice Hall, New Jersey. 
Heinz, M., S. Juranek and H. A. Rau, 2012, "Do women behave more reciprocally than men? Gender differences in real effort dictator games" Journal of Economic Behaviour \& Organization , v. 83, 195-110.

Kettner, S.L. and S. Ceccato, 2014. "Framing Matters in Gender-Paired Dictator Games" University of Heidelberg Discussion Papers.

Montagu, A. The Natural Superiority of Women, New York: Macmillan.

Rigdon M. and A. S. Levine, 2011, Gender, Expectations and the Price of Giving, SSRN working paper

Selten, R. and A. Ockenfels, 1998. "An Experimental Solidarity Game." Journal of Economic Behaviour and Organization, 34(4): 517-39.

Servatka M., 2009." Separating reputation, social influence, and identification effects in a dictator game". European Economic Review, 53, 197-209. 


\section{Appendix 1: \\ Dictators' Instructions (Session 1)}

Welcome to our experiment. Please do not communicate with other participants and think carefully before making your choice. The quality of our research depends on both aspects.

Upon arrival, you have been delivered an envelope with an identification number ( $\mathrm{A} 1, \mathrm{~A} 2$, etc.). Please remember this number: you will have to report it to the experimenters in order to collect your reward.

How does the experiment proceed?

You have found two sheets of papers, one with all the information for the experiment (page 1), one (page 2) where you just have to report your sex, crossing the right box ( $F, M)$.

The experimenters will give you a participation fee of 3 euro, which you will collect today when you leave. The participation fee will be given to all subjects who are here today (in both rooms).

However, an extra sum of money will be given only to subjects who are in this room, i.e.; only to A participants. The extra sum of money is expressed in experimental tokens, each worth 40 Euro cent (just to make things easy for our final report). In total, you are endowed with 20 tokens and now you have to decide how many tokens you want to keep for yourself and how many you want to transfer to a fellow participant who is now sitting in the adjacent room and who received the participation fee, but no money extra.

Once you decide how many tokens you want to send to this anonymous participant, please write the amount below:

I want to transfer:

tokens

Notice that, if you transfer 5 tokens, at the end of the experiment you have 15 tokens ( 6 euro) plus 3 euro participation fee and the other subject will have 3 euro plus 5 tokens you transferred to him ( 2 euro). Therefore, your final earnings will be 9 euro, his $\backslash$ her final earnings will be 5 euro.

Once you have completed the task, please put the sheets of paper back in the envelope and give it back to the monitor. The monitor will put all the envelopes in one big box, and together with the other's room monitor will form couples of subjects and the final earning will be calculated.

However, you will be not be paid today, but we will send you a mail with the indication of the day and the date in which you can collect the payments. You will just have to report your identification number and you will be paid at once. 